\title{
Quantifying fluorescence enhancement for slowly diffusing single molecules in plasmonic near fields
}

Martín Caldarola, Biswajit Pradhan, and Michel Orrit

Citation: The Journal of Chemical Physics 148, 123334 (2018); doi: 10.1063/1.5023171

View online: https://doi.org/10.1063/1.5023171

View Table of Contents: http://aip.scitation.org/toc/jcp/148/12

Published by the American Institute of Physics

\section{Articles you may be interested in}

A versatile optical microscope for time-dependent single-molecule and single-particle spectroscopy

The Journal of Chemical Physics 148, 123316 (2018); 10.1063/1.5009134

Two states or not two states: Single-molecule folding studies of protein L

The Journal of Chemical Physics 148, 123303 (2018); 10.1063/1.4997584

Kinetic analysis of single molecule FRET transitions without trajectories

The Journal of Chemical Physics 148, 123328 (2018); 10.1063/1.5006038

Solvent effect on FRET spectroscopic ruler

The Journal of Chemical Physics 148, 123331 (2018); 10.1063/1.5004205

Preface: Special Topic on Single-Molecule Biophysics

The Journal of Chemical Physics 148, 123001 (2018); 10.1063/1.5028275

Spectrally resolved single-molecule electrometry

The Journal of Chemical Physics 148, 123307 (2018); 10.1063/1.5008936

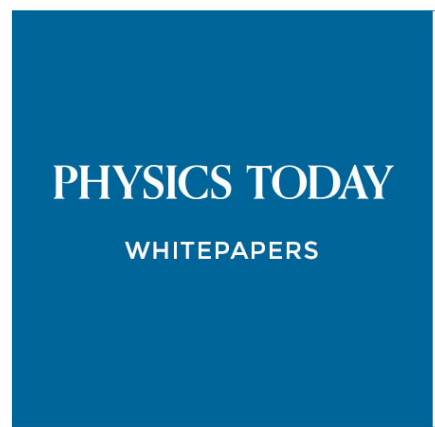

ADVANCED LIGHT CURE ADHESIVES

Take a closer look at what these environmentally friendly adhesive systems can do

\section{READ NOW}

PRESENTED BY

Q MASTERBOND 


\title{
Quantifying fluorescence enhancement for slowly diffusing single molecules in plasmonic near fields
}

\author{
Martín Caldarola, Biswajit Pradhan, and Michel Orrit ${ }^{\mathrm{a})}$ \\ Huygens-Kamerlingh Onnes Laboratory, Leiden University, 2300 RA Leiden, The Netherlands
}

(Received 22 January 2018; accepted 26 February 2018; published online 16 March 2018)

\begin{abstract}
Gold nanorods are extensively used for single-molecule fluorescence enhancement as they are easy to synthesize, bio-compatible, and provide high light confinement at their nanometer-sized tips. The current way to estimate fluorescence enhancement relies on binned time traces or on fluorescence correlation spectroscopy. We report on novel ways to extract the enhancement factor in a singlemolecule enhancement experiment, avoiding the arbitrary selection of one or a few high-intensity burst(s). These new estimates for the enhancement factor make use of the whole distribution of intensity bursts or of the interphoton delay distribution, which avoids the arbitrary binning of the fluorescence intensity time traces. We present experimental results on the bi-dimensional case, experimentally achieved using a lipid bilayer to support the diffusion of fluorophores. We support our findings with histograms of fluorescence bursts and with an analytical derivation of the interphoton delay distribution of (nearly) immobilized emitters from the fluorescence intensity profile. Published by AIP Publishing. https://doi.org/10.1063/1.5023171
\end{abstract}

\section{INTRODUCTION}

Single quantum emitters such as fluorophores, quantum dots, color centers, and fluorescent proteins have become powerful tools for modern science since they provide nanometersized probes that can be used to extract information about the local environment, ${ }^{1,2}$ oxidation state of molecules, ${ }^{3}$ and proximity of other emitters using Förster resonance energy transfer (FRET). ${ }^{4-6}$ These unique advantages are joined to those of non-invasive optical methods.

Fluorescence enhancement by plasmonic nanostructures has been successfully used in the past to increase the signal from weak emitters ${ }^{7-9}$ even in living cells. ${ }^{10}$ In a nutshell, fluorescence enhancement by metallic nanoparticles refers to a considerable increase in the rate of detected photons whenever a (often weakly) fluorescent molecule is placed in the vicinity of the nanoparticle. Enhancement heavily relies on the surface plasmon resonance of the nanoparticle, which often lies in the optical spectral range. When excited at this resonance frequency, the nanoparticles can concentrate optical fields in tiny volumes, the so-called "hot spots," providing a sub-diffraction working volume that can be exploited to extend the powerful technique fluorescence correlation spectroscopy (FCS) to micromolar concentrations. ${ }^{1-15}$ Notably, this approach was also used to study molecular diffusion in the membrane of a living cell. ${ }^{16}$ Fluorescence enhancement also provides a way to extend the powerful tools of single-molecule spectroscopy to weakly emitting species.

Regardless of the origin of the fluorescence photons, a usual approach is to record the arrival time of each individual photon in the so-called time-correlated single-photon counting (TCSPC) approach in a time-tagged time-resolved (TTTR)

a)Electronic mail: orrit@physics.leidenuniv.nl configuration. ${ }^{17,18}$ Thanks to the high-speed electronics and pulsed excitation sources available commercially, the absolute arrival times (also called macrotimes) can be determined with picosecond accuracy and, with the proper synchronization to the excitation source, the "nanotimes" 19 can be also determined. The nanotime is usually used to obtain the lifetime histogram, which can be used to gain insight into the underlying mechanism of the emission. For example, in the case of fluorescence, the radiative and non-radiative rates can be accessed experimentally with a measurement of the lifetime if the quantum yield is known. ${ }^{20}$ The output of a TTTR experiment can be represented as a classical function of time, ${ }^{21}$

$$
I(t)=\sum_{i} \delta\left(t-t_{i}\right)
$$

where $\delta(t)$ is the usual Dirac function and $t_{i}$ is the absolute arrival time for each detected photon in the experiment (measured relative to the start of the experiment, the macrotime). We shall call this function unbinned time trace.

In spite of the high temporal resolution provided by such experiments, the usual way to characterize the emission of quantum emitters is to display the time trace of the number of detected photons in a certain integration time (or binning time) and to complement this with a histogram of detected photon counts per time bin. Such a characterization inherently introduces an arbitrary parameter, the binning or integration time, that may bias the obtained distribution. ${ }^{21}$ Alternatively, correlation functions can be calculated from the unbinned time trace to exhibit the characteristic time of a process of interest, such as the diffusion ${ }^{22,23}$ or rotation $^{24}$ characteristic times of molecules in solution as well as other molecular properties even at single-molecule level. ${ }^{25,26}$ This approach can provide estimates of the enhancement factor, but is very sensitive to background corrections. ${ }^{27,28}$ The 
common practice to determine fluorescence enhancement is to screen the time trace for the strongest fluorescence burst(s), corresponding to unlikely event(s) that a molecule occupies the best position in the hot spot of the plasmonic near field for a long enough time. This procedure, often nicknamed "cherry-picking," depends on the binning time, on the diffusion constant, and on the stochastic character of each molecule's trajectory. There is no warranty that waiting for longer times, or binning at higher resolution, would not lead to larger enhancement factors. Therefore, there is a pressing need for less arbitrary ways to quantify the enhancement factor.

Here, we focus on the use of a less common quantity, the interphoton delay distribution $p(\tau)$, to characterize the emission of quantum emitters and to extract reliable information about an emitting system avoiding the introduction of any arbitrary binning time. The interphoton delay distribution expresses the delay distribution between consecutive photons: after each photon detection, the probability density to observe the next photon at a time $\tau$ is $p(\tau)(p(\tau) \geq 0) .{ }^{29}$ Experimentally, it can be obtained by simply plotting a histogram of the time differences between successively detected photons.

In this paper, we present a model to relate the interphoton delay distribution to the spatial distribution of fluorescence intensity delivered by a single quantum emitter in the limit of slow diffusion. We show that $p(\tau)$ encodes information about the intensity distribution inside the volume accessible to diffusers. We will illustrate this point with single-molecule fluorescence in a bi-dimensional case both with a Gaussian beam shape and with addition of a power-law model of enhanced fluorescence by a gold nanorod. Furthermore, we propose to use the interphoton delay distribution to estimate the enhancement factor in fluorescence enhancement experiments, avoiding arbitrary parameters such as a binning time.

This paper is organized as follows. First we present a theoretical derivation of the interphoton delay distribution in Sec. II. In Sec. III, we compare the experimental and theoretical results in the simple case of an emitter switching between two intensity levels. Then, in Sec. IV, we move to the more complex case of two-dimensional diffusion of molecules under excitation by a Gaussian beam, where our model captures the essence of the process. In Sec. V, we present a simplified model for the enhancement from a single nanoparticle, using the interphoton delay distribution to characterize the phenomena. Finally, in Sec. VI we analyze experimental data and compare enhancement factors obtained by the "cherry-picking" procedure and the other estimates provided by the interphoton delay histogram and by a statistical burst analysis.

\section{THEORETICAL FRAMEWORK}

We seek to relate the interphoton delay distribution in a fluorescence experiment with the spatial distribution of intensities used to excite the fluorescent molecules. The interphoton delay distribution defined above is represented by the probability density function $p(\tau)$. Thus the probability of detecting the next photon between times $\tau$ and $\tau+\mathrm{d} \tau$ is $p(\tau) \mathrm{d} \tau$ and the normalization condition holds: $\int_{0}^{\infty} p(\tau) \mathrm{d} \tau=1$.

Let us start from the simplest case of a constant detected intensity $w$ (in counts per second). Such a signal gives rise to exponentially distributed interphoton delay times, i.e.,

$$
p(\tau)=w \exp (-w \tau) .
$$

This is a direct consequence of the memory-free character of the photon emission, which leads to a Poisson distribution of the number of photons emitted per binning time and to an exponential distribution of interphoton delays. We note that this distribution can be obtained with a fluorescent molecule excited at a constant intensity, for example, a fixed molecule immobilized on a substrate.

Let us now consider the limit of very slow diffusion. Variations in the local intensity seen by an individual emitter are much slower than delays between photon detection events so that there arises a distribution of intensities $Q(w)$ corresponding to various spatial configurations of emitters in and around the excitation focal spot. Averaging over this distribution of intensities gives rise to the interphoton delay distribution $p(\tau)=\int_{0}^{\infty} w e^{-w \tau} Q(w) \mathrm{d} w$, which corresponds to over all possible intensities (rates) $w$ and can be rewritten as

$$
p(\tau)=-\frac{\mathrm{d}}{\mathrm{d} \tau} \mathscr{L}\{Q\}(\tau),
$$

where $\mathscr{L}\{Q\}(\tau)=\int_{0}^{\infty} e^{-w \tau} Q(w) \mathrm{d} w$ denotes the Laplace transform $^{30}$ of the function $Q(w)$. If we seek the interphoton delay distribution corresponding to the added signals of two sources with intensity distributions $P(w)$ and $Q(w)$, we can use the total intensity $T(w)$ that can be calculated as the sum of a combined probability of source 1 emitting at a rate $x$ and source 2 at a rate $(w-x)$, i.e., by convoluting the two intensity functions: $T(w)=\int P(x) Q(w-x) \mathrm{d} x$. Using the convolution theorem we can write the Laplace transform of the intensity distribution as the product of the Laplace transforms of the two distributions, from which we can deduce the interphoton delay distribution.

Let us now consider as a source one point-like emitter at position $\mathbf{r}$, for example, a fluorescent molecule diffusing around an optical intensity maximum. The detected intensity will generally be a product of the position-dependent local excitation intensity and of a position-dependent collection efficiency, with some molecular parameters involved in the fluorescence process (absorption cross section, fluorescence quantum yield, etc.). We write the product of these factors as a position-dependent fluorescence intensity profile $I(\mathbf{r})$.

At this point, we stress some important approximations in our model. The first one is to neglect rotational diffusion of the molecules, which is a good approximation if we study times that are much longer than the rotational diffusion time. For a typical dye molecule in water, the rotational time will be on the nanosecond time scale, the translational time in the focal volume about $100 \mu \mathrm{s},{ }^{20}$ and the average delay between detected photons ranges from $10 \mu$ s to $1 \mathrm{~ms}$. The second important approximation is to assume that translational diffusion is slow compared to the photon detection rate, i.e., the molecules are nearly fixed during the emission process. A complete 
theory relaxing these approximations would be much more complicated and exceeds the scope of this work. Further, we neglect such experimentally relevant phenomena as blinking and photobleaching to limit the number of parameters in our model.

Under the mentioned approximations, the photon distribution can be deduced from the intensity distribution. Exploration of the diffusion volume $V$ accessible to the moving emitter gives rise to the following distribution of intensities:

$$
Q_{1}(w)=\frac{1}{V} \int_{V} \delta(w-I(\mathbf{r})) \mathrm{d}^{3} r,
$$

where $\delta(x)$ represents the Dirac delta function. To calculate the interphoton delay distribution using Eq. (3), we need the Laplace transform of $Q_{1}(w)$,

$$
\begin{aligned}
\mathscr{L}\left\{Q_{1}\right\}(\tau) & =1-\lambda(\tau), \quad \text { where } \\
\lambda(\tau) & \equiv \frac{1}{V} \int_{V}\{1-\exp [-I(\mathbf{r}) \tau]\} \mathrm{d}^{3} r .
\end{aligned}
$$

Note that, for an intensity variation with a finite range around the center, for example, due to Gaussian illumination and/or collection, $\lambda(\tau)$ is a small quantity which tends to zero for a large diffusion volume $V$.

We now consider the case of many $(N)$ emitters with a concentration $C=N / V$ diffusing in a large volume. Using the argument presented before for two emitters, the addition of one emitter will modify the intensity distribution by convolution with the one-emitter distribution function $Q_{1}(\tau)$. Using the convolution theorem for the Laplace transform, we can write the Laplace transform for the $N+1$ diffusers as

$$
\mathscr{L}\left\{Q_{N+1}\right\}(\tau)=\mathscr{L}\left\{Q_{N}\right\}(\tau) \mathscr{L}\left\{Q_{1}\right\}(\tau),
$$

from which we deduce $\mathscr{L}\left\{Q_{N}\right\}(\tau)=\left[\mathscr{L}\left\{Q_{1}\right\}(\tau)\right]^{N}$. Now we apply the statistical method of Stoneham ${ }^{31,32}$ by letting number and volume tend to infinity keeping the ratio constant to match the concentration $C$, and obtain

$$
\ln \left[\mathscr{L}\left\{Q_{N}(w)\right\}(\tau)\right]=N \ln [1-\lambda(\tau)] \approx-C V \lambda(\tau) .
$$

Therefore, using this result in Eq. (3) together with the definition from Eq. (5), we find the histogram of interphoton delays for a concentration $C$ of emitters diffusing in a fluorescence intensity profile described by $I(\mathbf{r})$,

$$
p(\tau)=-\frac{\mathrm{d}}{\mathrm{d} \tau}\left[\exp \left(-C \int_{V}(1-\exp [-\tau I(\mathbf{r})]) \mathrm{d}^{3} \mathbf{r}\right)\right]
$$

This is a general result that allows us to calculate the interphoton delay histogram for a solution with a concentration $C$ of slowly freely diffusing objects in a fluorescence intensity profile $I(\mathbf{r})$. We note that the general result above fulfills the normalization condition for $p(\tau)$, as required for any probability density function.

The case of infinitely fast diffusion of the emitters is easily obtained by letting the concentration $C$ go to infinity and the intensity $I(\mathbf{r})$ vanish, while keeping their product, i.e., the total brightness per unit volume, constant. It is easily seen that the limit of Eq. (8) becomes a single exponential distribution with an average intensity $W$,

$$
W=C \int_{V} I(\mathbf{r}) \mathrm{d}^{3} \mathbf{r} .
$$

This result is easily interpreted: in the fast diffusing case, each volume element contributes a constant intensity. Because the total intensity is constant, there are no intensity fluctuations and the distribution of delays is single-exponential. In other words, deviations from an exponential distribution of delays characterize fluctuations in the fluorescence intensity, themselves related to fluctuations of the number of emitters in the excitation volume. Just as in FCS, these fluctuations become more and more important as the concentration is lowered.

\section{TWO-STATE EMITTER}

In order to show that we can avoid the binning of our TTTR data to extract valuable information about our experiment, we studied the simple case of an emitter switching between two fixed detected intensities. In such a scenario, and for slow enough switching, the interphoton delay distribution will be a bi-exponential function.

We experimentally access this situation by using fluorescence enhancement by individual gold nanorods. In this scenario, a 1000-fold intensity enhancement can be achieved for weak dyes. ${ }^{8,9}$ However, this enhancement value depends strongly on the position of the molecule in the nanoscale plasmonic hot spot of the structure, where the enhanced field is concentrated. Additionally, there is a competition between the emission enhancement and quenching effects, which become dominant at distances shorter than a few nm. ${ }^{9}$ Henceforth, we neglect quenching effects altogether. Thus the challenge in such experiments is to place the dye molecules in the desired position to achieve high enhancement values. An elegant solution to place the molecules in the desired position is the technique called transient binding. ${ }^{33}$ Briefly, we use two complementary single-stranded DNA sequences, one attached to the surface of a gold nanorod and the other, diffusing one, marked with a single Cy5 molecule (fluorescence quantum yield 0.27 ). The strand attached to the gold surface is called the docking strand, since it allows the complementary strand to dock in one specific site and the latter is called the imaging strand since it allows fluorescence detection.

This experimental scheme is shown in Fig. 1. We immobilized a gold nanorod on a glass surface and the imaging strands diffuse freely in the buffer solution. When an imaging strand diffuses close to the docking strand, the DNA can hybridize, forming a temporary double strand DNA. In that scenario, the single fluorescent label is placed in the plasmonic hot spot of the nanorod and gets enhanced, giving a higher signal than the other molecules in the detection volume. This approach allows us to put a single dye in a well-defined position in the near field of the nanorod for an exponentially distributed time whose mean value can be controlled with the length of the DNA and the environment conditions. ${ }^{34}$ Since the dye molecule is fixed at a certain point in space, with a fixed exciting intensity, we expect a fixed detected intensity. The main advantage of this experimental approach is that the hybridization is transient and after some time the DNA will de-hybridize, freeing the docking site for another imaging strand to come. As a result, photobleaching of the dye is 

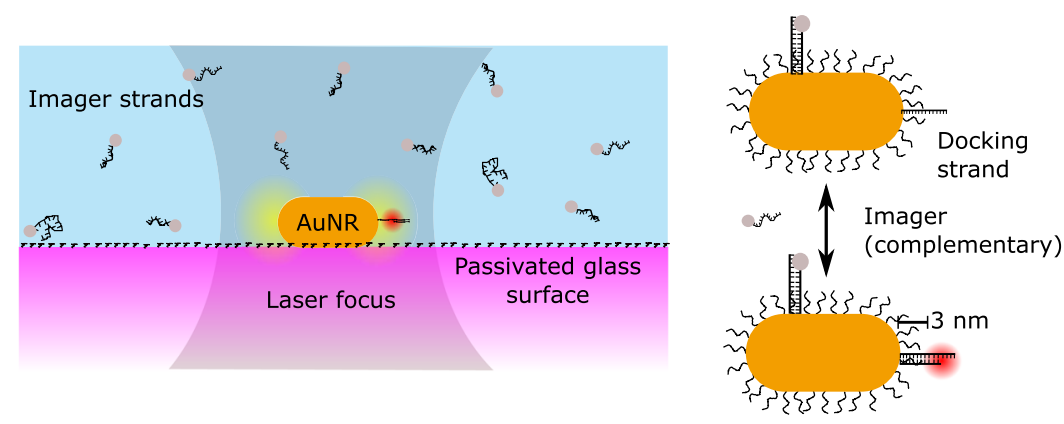

FIG. 1. Transient binding experimental scheme. We used a home-made confocal microscope to excite and detect the imaging strand-Cy5 constructs in the solution. The signal from the molecules in the solution is low, thus we enhance the fluorescence signal using immobilized gold nanorods (average size: $45 \times 90 \mathrm{~nm}$ ) on a glass surface. In order to experimentally access the same spatial position in the plasmonic hot spot, we use a transient binding technique with a 15-base pair DNA as the docking strand and a 10-base pair complementary labeled DNA as the imaging strand. The docking strand is attached to the gold nanorod surface using two thiol bonds.

not a limiting factor for the experiment, since the same point in space can be probed multiple times with different single molecules.

Figure 2 shows the experimental results on our twointensity system. On panel (a), on the top left, we show the typical histogram of number of photons per bin time, characterizing the binned fluorescence time trace of panel (b), which shows the fluorescence time trace. The solid lines in panel (a) are fits with two Poisson distributions. We attribute the deviation from the experimental histogram to additional experimental noise not included in the model. Two levels can be clearly identified. The high-fluorescence level corresponds to hybridized docking and imaging DNA strands so that a dye molecule is immobilized in the hot spot, emitting enhanced fluorescence. After some seconds, the DNA de-hybridizes either before or after bleaching of the dye, and the imaging strand leaves the hot spot. In both cases, the signal has vanished. The low-level signal corresponds to the luminescence of the gold nanorod itself and to the background fluorescence of diffusing and unenhanced molecules in the confocal volume. This system thus fulfills our purpose by providing a stream of detected photons with two well-defined intensity levels.
We would like to retrieve these two levels using the interphoton delay distribution by fitting a bi-exponential function. Figure 2(c) shows the experimental curve and the fitting, from which we extracted the two intensity levels marked in (a) and (b) with dashed lines. These levels clearly reproduce the levels evidenced by the binned time trace and intensity histograms, but they were obtained without the need of any arbitrary bin time.

The results in this simple case show how useful this type of analysis can be, since we were able to extract useful information from our experimental TTTR data without introducing any arbitrary parameter.

\section{SLOW DIFFUSION IN A 2D GAUSSIAN INTENSITY PROFILE}

A more interesting scenario to use our analysis is the problem of two-dimensional diffusion of fluorescent molecules in a Gaussian beam, described by an intensity function $I(\mathbf{r})=I_{0} \exp \left[-r^{2} / \sigma^{2}\right]$, where $(r, \theta)$ are the normal polar coordinates in the plane and $\sigma$ represents the waist of the beam. In this case, the intensity profile is bi-dimensional and only (a)

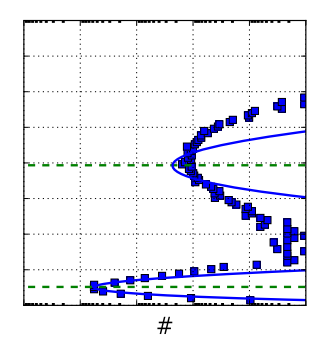

(b)

(c)

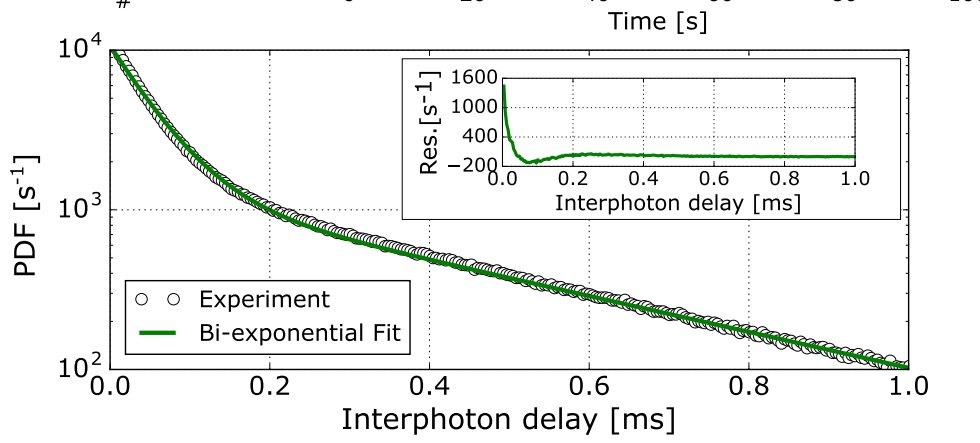

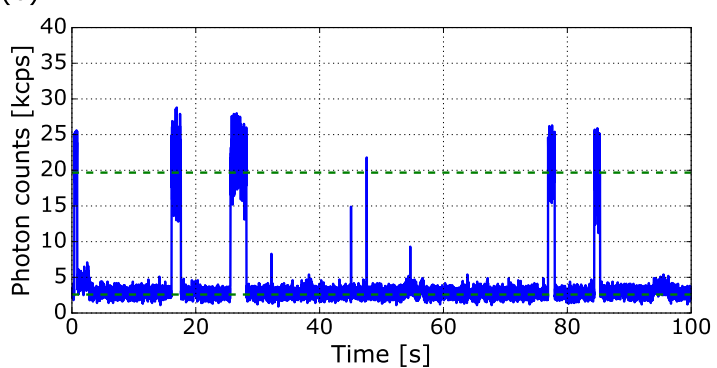

FIG. 2. Fluorescence enhancement of single Cy5 molecules with transient binding. (a) Binned intensity histogram (squares) with a fit with two Poisson distributions (blue lines). (b) Binned fluorescence time trace (bin time $=10 \mathrm{~ms}$ ). From these two plots, two intensity levels are clearly recognized: the high level corresponds to the enhanced fluorescence signal of one Cy5 molecule at a fixed position in the hot spot while the low level corresponds to the intensity from gold nanorod luminescence plus the contribution of all diffusing molecules (enhanced and unenhanced) in the detection volume. (c) Interphoton delay probability density function (PDF) obtained from the TTTR measurements (empty dots) and a biexponential fit, one for each intensity level. The retrieved intensities from this fit are shown in green dashed lines in (a) and (b), and coincide with the high and low fluorescence levels in the time trace. The inset shows the residuals for the fit. 
(a)
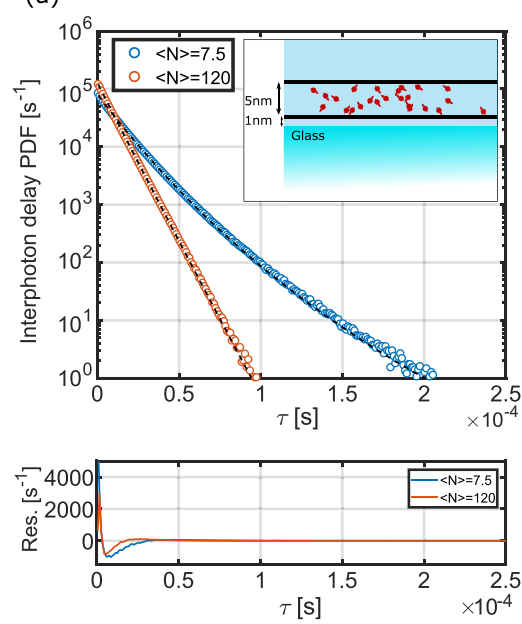

(b)
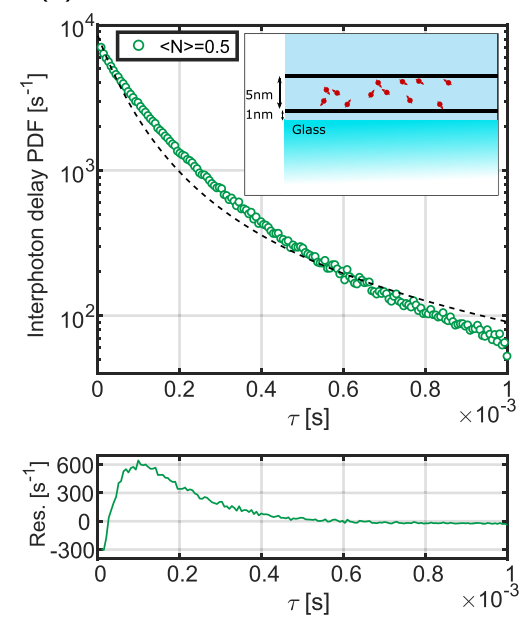

FIG. 3. Bidimensional molecular diffusion probed with a Gaussian beam. (a) Interphoton delay probability density function (PDF) for the case of high concentrations of molecules, with an average number of molecules $\langle N\rangle=7.5$ or 120 in the detection area $A=\pi \sigma^{2}$ (corresponding to approximately 14 and 225 molecules $/ \mu \mathrm{m}^{2}$, respectively). The circles are experimental values while the dashed lines are fits with the model from Eq. (10). (b) Interphoton delay probability density function for the case of a low concentration of molecules, $\langle N\rangle=0.5$ (approximately 0.9 molecules $/ \mu \mathrm{m}^{2}$ ). In this case, we find clear deviations from our model of slow diffusion, possibly indicating averaging of number fluctuations by diffusion, and a more exponential-like decay. The insets show a scheme of the experimental configuration of the lipid bi-layer on the glass surface with the typical dimensions involved (not to scale). In the bottom panel, we show the residuals for the respective fits maintaining the color code.

depends on the distance to the center of the beam. We take a concentration $C$ of fluorescent molecules per unit area. Using this intensity distribution in Eq. (8), we calculate the expected interphoton delay distribution $p_{G}(\tau)$ and find the integral form

$$
p_{G}(\tau)=-\frac{\mathrm{d}}{\mathrm{d} \tau}\left[\exp \left(-C \sigma^{2} \pi \int_{\epsilon}^{1}\left(1-\exp \left[-\tau I_{0} u\right]\right) \frac{\mathrm{d} u}{u}\right)\right],
$$

where $\epsilon \equiv \exp \left(-\frac{R^{2}}{\sigma^{2}}\right)$ and $R$ is the maximum radius accessible to the diffusing molecules ( $\pi R^{2}$ is the area of the sample). Because of the smooth variation of the Gaussian beam, many single-molecule bursts have the same maximum intensity. Therefore, FCS provides a good estimate of single-molecule brightness. The case of enhanced fluorescence discussed in Sec. $\mathrm{V}$ is much more difficult to address by FCS. ${ }^{28}$

We studied the Gaussian case experimentally in a regular confocal microscope by confining the diffusion of ATTO647N dye molecules in a lipid bilayer, obtaining a two-dimensional case and a reduced diffusion coefficient of $D=4.4 \mu \mathrm{m}^{2} \mathrm{~s}^{-1}$, as presented previously. ${ }^{27}$

We performed the experiment for high and low concentrations of molecules in the lipid bilayer and fitted the experimental interphoton curves with $p_{G}(\tau)$ from Eq. (10) using the experimental value for the beam waist, $\sigma=292 \mathrm{~nm}$. Figure 3 shows the experimentally obtained interphoton delay probability density function for high (a) and low (b) concentrations along with the corresponding fits using our theoretical result. We note that the higher the concentration, the more the delay distribution resembles a single exponential. Indeed, a single exponential is expected in the limit of extremely high concentrations, where fluctuations $\Delta N$ of the number of molecules $\langle N\rangle$ in the Gaussian area become negligible, leading to a constant detected intensity and therefore to a single-exponential interphoton delay distribution.
A closer look at the curves in Fig. 3 reveals that the case of high concentration is well captured with our model while the low-concentration case is not. This is a direct consequence of the main approximation in our model that largely neglects the effect of diffusion. Another phenomenon we have ignored is photobleaching of the molecules in the laser beam. These deviations from our model could thus be studied through the interphoton delay distribution.

\section{INTERPHOTON DELAY DISTRIBUTION WITH FLUORESCENCE ENHANCEMENT}

We now turn to the case of plasmonic enhancement by an individual nanoparticle. The spatial distribution of fluorescence enhancement by a plasmonic structure is complex and depends on many parameters. For simplicity's sake, we model this distribution as a spherically symmetric profile around a spherical nanoparticle. The fluorescence intensity profile is taken as the sum of a Gaussian confocal volume similar to that of Sec. IV and of a near-field component modeled as a steeply decaying power law of radius,

$$
I(r)=W_{0}\left[\exp \left(-\frac{r^{2}}{\sigma^{2}}\right)+E\left(\frac{R_{N P}}{r}\right)^{\alpha}\right], \quad r \geq R_{\mathrm{NP}},
$$

where $W_{0}$ is the unenhanced intensity, $\sigma$ is the width of the Gaussian illumination, $E$ is the maximum enhancement factor at or close to the particle's surface, and $R_{\mathrm{NP}}$ is the nanoparticle radius. The exponent $\alpha$ is a free parameter used to simulate the short-range variation of the near field. For pure excitation enhancement by an electrostatic dipole field $\alpha=6$, for combined excitation and radiative enhancements of an electrostatic dipole field, we would have $\alpha=12$. We note that to recover the Gaussian case studied in Sec. IV, we need to take $R_{\mathrm{NP}}=0$. In order to obtain $p(\tau)$, we insert Eq. (11) into Eq. (8) and numerically solve the integral for $R_{\mathrm{NP}}=25 \mathrm{~nm}$. Equation (11) is a phenomenological expression meant to 
(a)

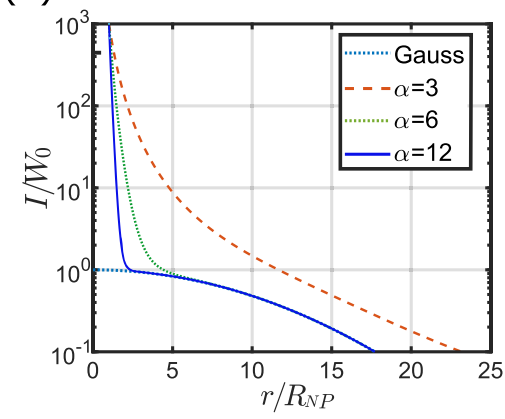

(b)

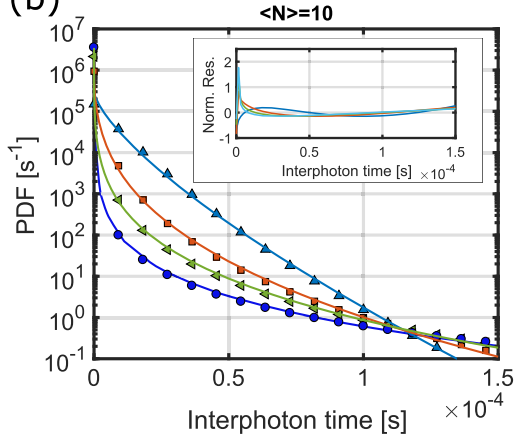

(c)

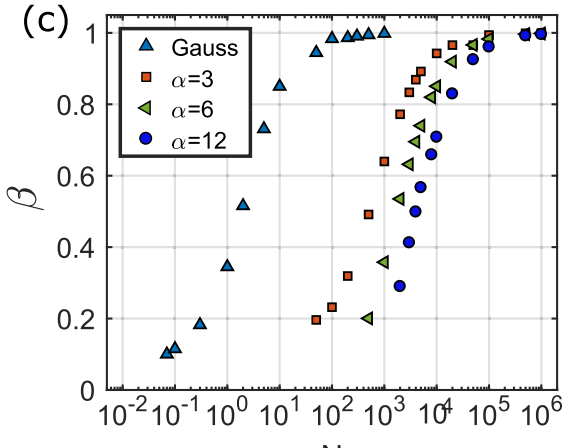

$<\mathrm{N}>$
FIG. 4. Simple model for plasmonic enhancement in two dimensions. (a) Normalized radial intensity distributions for Gaussian (blue dotted line) and near field model with $\alpha=3$ (red dashed line), $\alpha=6$ (green dotted line), and $\alpha$ $=12$ (blue solid line). For all the cases, the unenhanced intensity is $W_{0}=1.5 \times 10^{4} \mathrm{cps}$ and for the enhanced case we used $R_{\mathrm{NP}}=25 \mathrm{~nm}$ and $E=1000$. (b) Interphoton delay probability density function for the three cases presented in (a) for a mean number of molecules $\langle N\rangle=10$ in the Gaussian area. The symbols present the data from the numerical evaluation of $p(\tau)$ and the solid lines are fits with stretched exponentials. We intentionally reduced the density of points for display. The inset shows the normalized residuals from the fits. (c) Extracted $\beta$ from the stretched exponential fit as a function of the mean number of molecules in the Gaussian area. The color code is maintained throughout the whole figure. explore the effect of the near field. It completely ignores quenching, polarization, and the complex spatial structure of the near field.

Figure 4(a) shows the comparison of the spatial intensity distribution for three different values of the exponent $\alpha$ with the unenhanced case. With these intensity spatial distributions, we calculated the interphoton delay distribution by numerically solving the integral in Eq. (8). Note that the area inside the nanoparticle is not accessible to the molecules so the integration was carried for $r \geq R_{\mathrm{NP}}$. The obtained distributions are shown in Fig. 4(b) for the case of $\langle N\rangle=10$ molecules in the Gaussian area $\left(A=\pi \sigma^{2}\right)$. Clearly the distributions with enhancement show more probability density at extremely short interphoton times, corresponding to the high emission rates produced by molecules occasionally entering the near-field area with high enhanced intensities. These events correspond to bright bursts in the fluorescence time trace. The lower the concentration of molecules, the more seldom these events will be, and the further away the delay distribution will be from a single exponential.

In order to qualitatively compare the interphoton delay distributions for the different cases, we decided to fit them with stretched exponentials

$$
f(\tau)=A \exp \left[-(\lambda \tau)^{\beta}\right]
$$

to characterize the deviation of the interphoton delay distribution from a single exponential. As we mentioned before, in the case of very high molecular concentrations we expect to recover $\beta=1$, i.e., an exponential behavior, whereas large number fluctuations will give rise to strong deviations from single exponential and to a smaller stretching exponent. The empiric fit function in Eq. (12) works reasonably well for short times, but fails to reproduce the long-time tails of the delay distribution. Therefore, we focus our analysis on the short-time domain, which contains the most useful information about plasmonic enhancement.

We fitted the calculated probability density functions for different concentrations of molecules ranging from a very diluted sample ( 1 molecule in the Gaussian area, $3 \times 10^{-3}$ in the near-field area) to an extremely high number of molecules $\left(10^{6}\right.$ in the Gaussian area, $3 \times 10^{3}$ in the near field). Figure 4(c) shows the obtained stretching exponent $\beta$ as a function of concentration and for the Gaussian beam and the enhanced case with three different exponents $\alpha=3,6$, and 12. In the Gaussian case, we obtain an exponential behavior, characterized with $\beta=1$ only for $\langle N\rangle \geq 100$. This corresponds to the situation when number fluctuations in the detection area are negligible and thus a nearly constant detected intensity is obtained.

To obtain a single-exponential interphoton delay distribution in the enhanced case, we should reach the high-density regime mentioned above, but considering the near-field area. Since the ratio of the near-field area $A_{\mathrm{NF}}$ [considered as the area that contains intensities higher than $\left.E W_{0} \exp (-1)\right]$ and the far-field area $A_{\mathrm{FF}}=\pi \sigma^{2}$ is $\frac{A_{\mathrm{NF}}}{A_{\mathrm{FF}}} \sim 3 \times 10^{-3}$, we roughly expect a difference of 3 orders of magnitude in the number of molecules needed to reach the single-exponential limit. Indeed, this is what our curves show, where for the enhanced case we approach $\beta=1$ around $\langle N\rangle=10^{5}$.

From Fig. 4(c), we see that the stretching exponent steeply decreases with concentration, as expected from the qualitative discussion above. This effect is more pronounced for a steeper decay of the fluorescence intensity profile, i.e., for larger values of $\alpha$. For very small concentrations, the stretched-exponential fit becomes poor, and the associated beta values have been omitted. We also note that the slope of the variation of $\beta$ with concentration is not very sensitive to the near-field decay characterized by $\alpha$ [see Fig. 4(c)]. 


\section{FLUORESCENCE TIME TRACES WITH ENHANCEMENT BY A GOLD NANOROD}

We now turn to results of an experiment with configuration similar to the one presented in Sec. IV. A Gaussian beam is focused on a single gold nanorod immobilized on a glass substrate, and dye molecules diffuse in a lipid bi-layer deposited on the same substrate, similar to Ref. 27. A fluorescence signal is produced whenever a dye molecule enters the diffraction-limited Gaussian beam, and an enhanced fluorescence signal appears if the molecule then enters either of the plasmonic hot spots around the tips of the gold nanorod. In order to obtain the enhancement value, we need to compare the detected intensity from a single-molecule enhancement event with the unenhanced intensity detected using the same experimental conditions, which was $100 \mathrm{kcps} .{ }^{27}$

Figure 5 presents the TTTR experimental data obtained in such an experiment in different ways, for two different gold nanorods. In the top panel, we have the bin-dependent time traces and burst histograms (binning time $1 \mathrm{~ms}$ ) while the lower panel shows the "bin-free" interphoton delay histograms. If we analyze the binned time traces in Figs. 5(a) and 5(c) and the insets, we observe that they present a qualitatively similar behavior: there is a constant baseline intensity, long weak bursts corresponding to molecules exploring the far field area and occasionally intense but short bursts corresponding to diffusion in the near-field area. ${ }^{27}$ The black crosses in the time traces indicate the highest burst found in that trace, which leads to "cherry picking" enhancements factors $E^{(C P)}=(4.5 \pm 1.5)$ and $E^{(C P)}=(2.3 \pm 0.7)$.

Another useful way to characterize the experimental enhancement is the histogram of binned intensities, as shown in Figs. 5(b) and 5(d). These histograms show a rapidly decaying tail where the characteristic decay intensity is larger for the top nanorod, indicating stronger enhancement. The steep decay of these histograms can be qualitatively understood by recalling the spatial distribution of near-field intensity at resonance, which is extremely high (around 300 times the incident intensity) very close to the tips and decays rapidly with distance to the tips. Therefore, there is a low probability for a diffusing molecule to reach this small area. For small distances, this probability density goes linearly with distance in the bi-dimensional case and as the squared distance in the three-dimensional case.

We analyzed the intensity distribution around a rod (25 $\times 47 \mathrm{~nm}^{2}$ ), calculated from a discrete-dipole approximation. ${ }^{9}$ In the 2D case, we found an approximate power law distribution with exponent 1.37. However, the tail of the experimental distribution is exponential, as can be seen in Figs. 5(b) and 5(d). There are several possible sources for such a discrepancy, such as the effect of photobleaching, the dead time of the detectors, and possibly the role of diffusion during the burst. Confirming the role of each of these effects would require full numerical calculations, with an accurate description of these effects. However, this is a complex and computationally extensive problem that is out of the scope of this paper.

Regardless of the reason for this deviation, we may empirically estimate the maximum enhancement through the histogram. Note that stronger enhancement corresponds to a broader histogram, with cutoff for larger photon number. If we model the tail of the normalized histogram decay as a single exponential $P(N)=M \exp \left(-N / N_{0}\right)$, we can estimate the maximum number of photons in the strongest burst by solving $P\left(N_{c}\right)=\varepsilon$ with $\varepsilon=1 / N_{\text {bins }}$, where $N_{\text {bins }}$ is the total number of bins in the time trace, here about $10^{6}$. This estimate roughly corresponds to a probability equal to unity of observing such an intense burst in the time trace. We thus obtain $N_{c}=N_{0} \ln (M / \varepsilon)$. For a typical value of $M \sim \times 10^{-3}$, we find $6.9 N_{0}$. With this method we obtain $E^{(H)}=(4.9 \pm 0.2)$

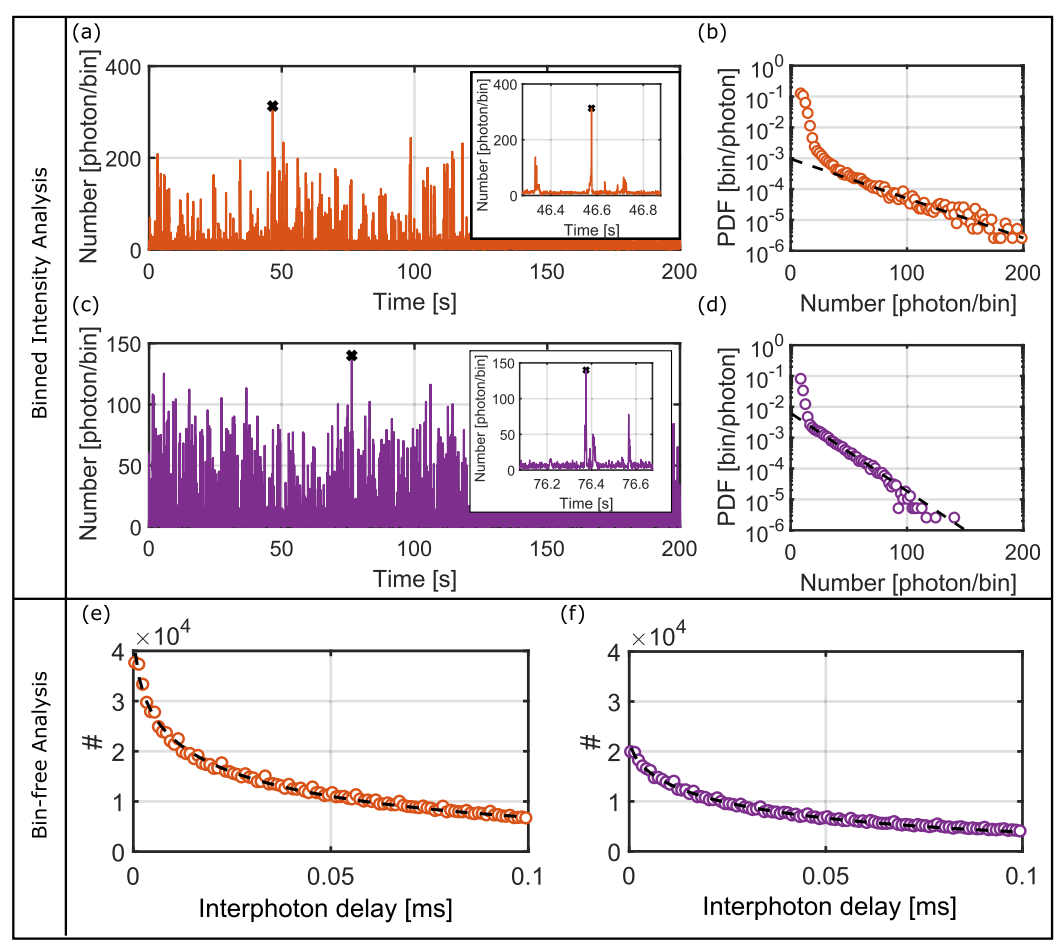

FIG. 5. Single-molecule fluorescence enhancement by gold nanorods. The top panel shows the typical binnedintensity plots while the bottom panel shows the bin-free interphoton histograms. [(a) and (c)] 1 ms-binned fluorescence intensity as a function of time for two individual nanorods. The nanorod in (a) presents a higher enhancement factor. The black cross in the plot indicates the maximum recorded intensity. The insets show respective zooms around the maximum intensity bursts. [(b) and (d)] Burst intensity histograms showing number of photons per $1 \mathrm{~ms}$-time bin for the traces in (a) and (c), respectively. The dashed black lines are exponential fits to the tail of the distributions. [(e) and (f)] Interphoton delay probability distributions for the experimental traces shown above. We also show a fit to the curves using a stretched-exponential model. The curves in purple and orange correspond to traces (c) and (a), respectively, and this color code is maintained throughout the whole figure. 
and $E^{(H)}=(2.40 \pm 0.05)$ for the plotted data in Figs. 5(b) and $5(\mathrm{~d})$.

Enhancement factors could also be extracted from an FCS analysis. ${ }^{27,28,35}$ However, this technique is very sensitive to background corrections and the relation between enhancement factor and correlation contrast is model-dependent.

We can also estimate the enhancement factor from the interphoton delay histograms. These histograms are shown for the same two nanorods in Figs. 5(e) and 5(f). Deviations from single-exponentials are clearly seen. To characterize them, we fit the histograms with a stretched exponential of time [see Eq. (12)], which involves only three parameters, against four for a bi-exponential decay. The maximum enhanced intensity is deduced from the slope of the histogram at the shorter measured interphoton time (200 ns) and provides another estimate of the enhancement factor based on a statistical quantity. Figures 5(e) and 5(f) show that larger enhancements correspond to a larger deviation of the histogram from a single exponential and to a larger slope at the smallest bin time of the histogram. With this procedure, we obtained an enhancement value of $E^{(I)}=(4.3 \pm 0.2)$ and $E^{(I)}=(2.0 \pm 0.1)$ for the presented nanorods.

We now compare the maximum intensities and the associated enhancement factors obtained for five different nanorods, for the whole $200 \mathrm{~s}$-long time trace, and after splitting each trace into 5 sub-traces of $40 \mathrm{~s}$ each. Figure 6 shows the example of the binned time trace (a), burst histogram (b), and the interphoton delay histogram (c) for the whole trace and for each sub-trace.

The associated results for the different methods are correlated in Figs. 6(c) and 6(d). The different symbols correspond to different nanorods and the colors refer to the different sub-traces for each rod. In Fig. 6(d), we observe an excellent correlation between the cherry-picking method and the statistical method based on the burst intensity histograms. Note that the same symbols are clustered together, showing that the enhancement factor obtained using different sub-traces with both methods lead to similar results. We also correlated the results from the interphoton delay distribution with the burst intensity histograms in Fig. 6(e), where a satisfactory correlation is found and the obtained enhancement factors are consistent with both other methods. However, the enhancement factors deduced from interphoton delay distributions present more dispersion than the other two.

Figure 6 confirms that the estimates of the enhancement factor deduced by three very different methods (time trace, burst histogram, and interphoton delays) are similar in value and consistent with one another. The values deduced from the cherry-picking procedure are surprisingly stable and reliable. They agree well with extrapolations of burst histograms fitted with a single-exponential function, although the justification for this analytical form is still missing. Unexpectedly, the enhancement factor deduced from the interphoton histogram

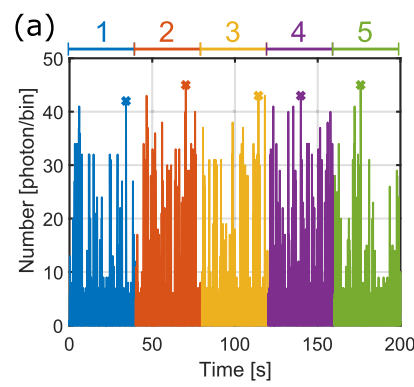

(d)

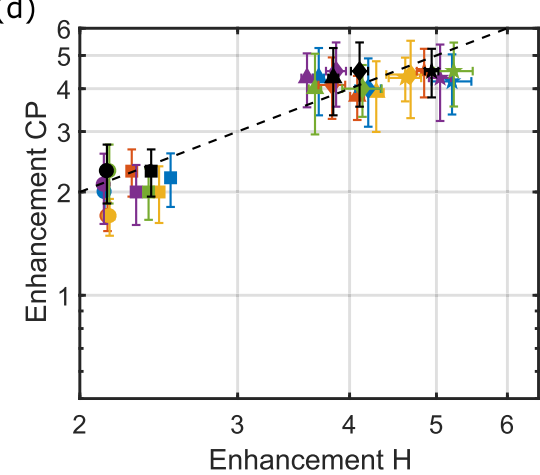

(b)

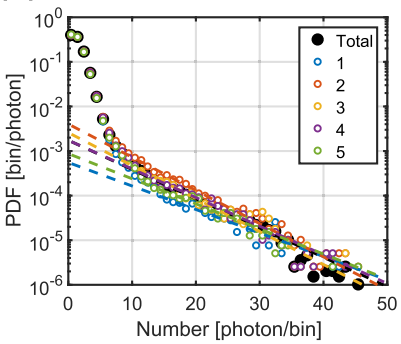

(c)

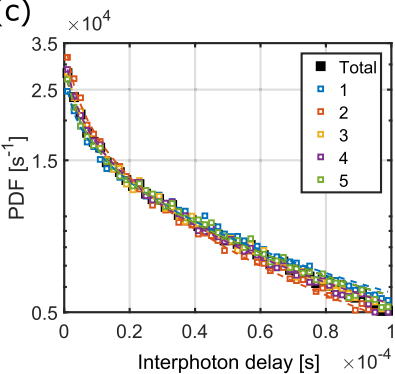

(e)

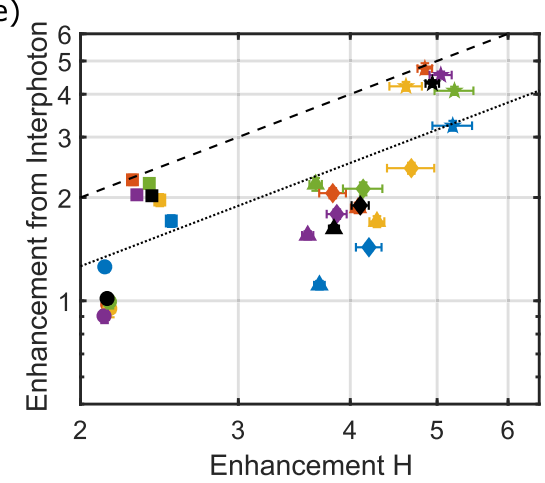

FIG. 6. Comparison of the enhancement factors obtained with different methods. (a) Binned time traces split into five different sub-traces of $40 \mathrm{~s}$ each (binning time $100 \mu \mathrm{s}$ ). The crosses show the maximum number of counts per bin obtained in each sub-trace, used to calculate the "cherry-picking" enhancement factor $E^{(C P)}$. At the top we show the length of the sub-traces and assign a number for reference. (b) Normalized burst histogram in number of photons per bin obtained for each of the sub-traces presented in (a). We also show in dashed lines the exponential fits to the tail of the probability density. The burst histogram for the total trace is plotted as well. (c) Interphoton delay distributions for the sub-traces and the total trace with their stretched-exponential fits. (d) and (e) show scatter plots of enhancement factors $E^{(C P)}, E^{(H)}, E^{(I)}$ deduced by the three methods. The different symbols correspond to different nanorods and the colors refer to the sub-traces used to obtain the enhancement factor. In black we show the results for the total trace. (d) Correlation between $E^{(C P)}$ from cherry picking and $E^{(H)}$ from burst histogram, showing an excellent correlation with slope equal to unity (dashed black line). (e) Correlation between $E^{(I)}$ from interphoton delay histograms and $E^{(H)}$ (the dotted line is a linear fit with slope 1). Here, we observe more dispersion of the data. 
appears to be the most sensitive to statistical fluctuations. This can be a consequence of fitting with a stretched exponential, which is an approximation. However, this method has the unique advantage that it can be applied to the experimental data directly, without any need for binning or other arbitrary parameters.

\section{CONCLUSIONS}

In this paper, we characterized single-molecule fluorescence traces obtained with a time-tagged time-resolved setup in a variety of experimental conditions with the interphoton delay distribution. This avoids the introduction of an arbitrary binning time.

We presented a theoretical treatment for the case of nearly static, slowly diffusing molecules that relates the interphoton delay distribution to the spatial intensity distribution explored by the molecules. With this model, we could reproduce the simple case of switching between two states with different intensities. We also explored the problem of molecules diffusing in two dimensions in a Gaussian beam. Our nearly static model works well at high concentrations, but shows deviations at lower concentrations, which may arise from diffusion or from other experimental deviations from the model.

Furthermore, we used the interphoton delay distribution to measure the fluorescence enhancement factor by individual gold nanorods. For our experimental traces with moderate enhancements, we obtained enhancement factors that are consistent with the accepted methods in the community with the advantage of avoiding the introduction of any arbitrary parameter that may influence the results. In the future, we plan to perform similar comparisons for the very large enhancement factors obtained with weakly emitting dyes.

\section{ACKNOWLEDGMENTS}

We would like to acknowledge financial support from NWO, the Netherlands Organization for Scientific Research, Grant No. ECHO 712.013.003 and the NanoFront program.

${ }^{1}$ W. Moerner and M. Orrit, "Illuminating single molecules in condensed matter," Science 283(5408), 1670-1676 (1999).

${ }^{2}$ F. Kulzer, T. Xia, and M. Orrit, "Single molecules as optical nanoprobes for soft and complex matter," Angew. Chem., Int. Ed. 49(5), 854-866 (2010).

${ }^{3}$ W. Zhang, M. Caldarola, B. Pradhan, and M. Orrit, "Gold nanorod enhanced fluorescence enables single-molecule electrochemistry of methylene blue," Angew. Chem., Int. Ed. 56(13), 3566-3569 (2017).

${ }^{4}$ E. A. Jares-Erijman and T. M. Jovin, "Fret imaging," Nat. Biotechnol. 21(11), 1387-1395 (2003).

${ }^{5}$ I. H. Stein, V. Schüller, P. Böhm, P. Tinnefeld, and T. Liedl, "Single-molecule FRET ruler based on rigid DNA origami blocks," ChemPhysChem 12(3), 689-695 (2011).

${ }^{6}$ S. Kalinin, T. Peulen, S. Sindbert, P. J. Rothwell, S. Berger, T. Restle, R. S. Goody, H. Gohlke, and C. A. Seidel, "A toolkit and benchmark study for FRET-restrained high-precision structural modeling," Nat. Methods 9(12), 1218-1225 (2012).

${ }^{7}$ A. Kinkhabwala, Z. Yu, S. Fan, Y. Avlasevich, K. Müllen, and W. Moerner, "Large single-molecule fluorescence enhancements produced by a bowtie nanoantenna," Nat. Photonics 3(11), 654-657 (2009).
${ }^{8}$ H. Yuan, S. Khatua, P. Zijlstra, M. Yorulmaz, and M. Orrit, "Thousand-fold enhancement of single-molecule fluorescence near a single gold nanorod," Angew. Chem., Int. Ed. 52(4), 1217-1221 (2013).

${ }^{9}$ S. Khatua, P. M. Paulo, H. Yuan, A. Gupta, P. Zijlstra, and M. Orrit, "Resonant plasmonic enhancement of single-molecule fluorescence by individual gold nanorods," ACS Nano 8(5), 4440-4449 (2014).

${ }^{10}$ T. S. van Zanten, M. J. Lopez-Bosque, and M. F. Garcia-Parajo, "Imaging individual proteins and nanodomains on intact cell membranes with a probebased optical antenna," Small 6(2), 270-275 (2010).

${ }^{11}$ L. C. Estrada, P. F. Aramendía, and O. E. Martínez, "10 000 times volume reduction for fluorescence correlation spectroscopy using nano-antennas," Opt. Express 16(25), 20597-20602 (2008).

${ }^{12}$ C. Manzo, T. S. van Zanten, and M. F. Garcia-Parajo, "Nanoscale fluorescence correlation spectroscopy on intact living cell membranes with NSOM probes,” Biophys. J. 100(2), L8-L10 (2011).

${ }^{13}$ A. A. Kinkhabwala, Z. Yu, S. Fan, and W. Moerner, "Fluorescence correlation spectroscopy at high concentrations using gold bowtie nanoantennas," Chem. Phys. 406, 3-8 (2012).

${ }^{14}$ D. Punj, J. de Torres, H. Rigneault, and J. Wenger, "Gold nanoparticles for enhanced single molecule fluorescence analysis at micromolar concentration," Opt. Express 21(22), 27338-27343 (2013).

${ }^{15}$ S. Khatua, H. Yuan, and M. Orrit, "Enhanced-fluorescence correlation spectroscopy at micro-molar dye concentration around a single gold nanorod," Phys. Chem. Chem. Phys. 17(33), 21127-21132 (2015).

${ }^{16}$ V. Flauraud, T. S. van Zanten, M. Mivelle, C. Manzo, M. F. Parajo, and J. Brugger, "Large-scale arrays of bowtie nanoaperture antennas for nanoscale dynamics in living cell membranes," Nano Lett. 15(6), 4176-4182 (2015).

${ }^{17}$ M. Wahl, Time-Correlated Single Photon Counting, Technical report, (PicoQuant, Berlin, Germany, 2014); see http://www.picoquant.com/images/ uploads/page/files/7253/technote_tcspc.pdf.

${ }^{18}$ W. Becker, Advanced Time-Correlated Single Photon Counting Applications, Springer Series in Chemical Physics (Springer, 2015), Vol. 111.

${ }^{19}$ The usual denomination is microtime but we opt for nanotime to avoid confusion with the macrotime.

${ }^{20}$ J. Lakowicz, Principles of Fluorescence Spectroscopy (Springer US, 2007).

${ }^{21}$ M. Lippitz, F. Kulzer, and M. Orrit, "Statistical evaluation of single nanoobject fluorescence," ChemPhysChem 6(5), 770-789 (2005).

${ }^{22}$ D. Magde, E. Elson, and W. W. Webb, "Thermodynamic fluctuations in a reacting system-Measurement by fluorescence correlation spectroscopy," Phys. Rev. Lett. 29(11), 705 (1972).

${ }^{23}$ E. Haustein and P. Schwille, "Fluorescence correlation spectroscopy: Novel variations of an established technique," Annu. Rev. Biophys. Biomol. Struct. 36, 151-169 (2007).

${ }^{24}$ A. Loman, I. Gregor, C. Stutz, M. Mund, and J. Enderlein, "Measuring rotational diffusion of macromolecules by fluorescence correlation spectroscopy," Photochem. Photobiol. Sci. 9(5), 627-636 (2010).

${ }^{25}$ M. A. Medina and P. Schwille, "Fluorescence correlation spectroscopy for the detection and study of single molecules in biology," BioEssays 24(8), 758-764 (2002).

${ }^{26}$ A. Ghosh, S. Isbaner, M. Veiga-Gutiérrez, I. Gregor, J. Enderlein, and N. Karedla, "Quantifying microsecond transition times using fluorescence lifetime correlation spectroscopy," J. Phys. Chem. Lett. 8(24), 6022-6028 (2017).

${ }^{27}$ B. Pradhan, S. Khatua, A. Gupta, T. Aartsma, G. Canters, and M. Orrit, "Gold-nanorod-enhanced fluorescence correlation spectroscopy of fluorophores with high quantum yield in lipid bilayers," J. Phys. Chem. C 120, 25996-26003 (2016).

${ }^{28}$ L. Langguth and A. F. Koenderink, "Simple model for plasmon enhanced fluorescence correlation spectroscopy,” Opt. Express 22(13), 15397-15409 (2014).

${ }^{29} \mathrm{R}$. Verberk and M. Orrit, "Photon statistics in the fluorescence of single molecules and nanocrystals: Correlation functions versus distributions of on- and off-times," J. Chem. Phys. 119(4), 2214 (2003).

${ }^{30}$ Note that the Laplace transformation is usually applied to time-dependent functions, giving rate-dependent functions. Here, we apply it to a function of the rate and thus obtain a time-dependent transform.

${ }^{31}$ A. M. Stoneham, "Shapes of inhomogeneously broadened resonance lines in solids," Rev. Mod. Phys. 41, 82-108 (1969).

${ }^{32}$ L. Fleury, A. Zumbusch, M. Orrit, R. Brown, and J. Bernard, "Spectral diffusion and individual two-level systems probed by fluorescence of single terrylene molecules in a polyethylene matrix," J. Lumin. 56(1-6), 15-28 (1993). 
${ }^{33}$ G. Acuna, F. Möller, P. Holzmeister, S. Beater, B. Lalkens, and P. Tinnefeld, "Fluorescence enhancement at docking sites of DNAdirected self-assembled nanoantennas," Science 338(6106), 506-510 (2012).

${ }^{34}$ R. Jungmann, C. Steinhauer, M. Scheible, A. Kuzyk, P. Tinnefeld, and F. C. Simmel, "Single-molecule kinetics and super-resolution microscopy by fluorescence imaging of transient binding on DNA origami," Nano Lett. 10(11), 4756-4761 (2010).

${ }^{35}$ D. Punj, M. Mivelle, S. B. Moparthi, T. S. van Zanten, H. Rigneault, N. F. van Hulst, M. F. García-Parajó, and J. Wenger, "A plasmonic 'antennain-box' platform for enhanced single-molecule analysis at micromolar concentrations," Nat. Nanotechnol. 8(7), 512 (2013). 